\title{
The newly revised Interview for Deteriorations in Daily Living Activities in Dementia (R-IDDD2): Distinguishing initiative from performance at assessment
}

Clarissa M. Giebel ${ }^{1,2,}{ }^{*}$, David Challis ${ }^{3}$, and Daniela Montaldi ${ }^{2}$

${ }^{1}$ School of Health Sciences, University of East Anglia, UK

2 Division of Neuroscience and Experimental Psychology, University of Manchester, UK

${ }^{3}$ Personal Social Services Research Unit, The University of Manchester, UK

${ }^{*}$ Correspondence should be addressed to: Clarissa Giebel, School of Health Sciences, University of East Anglia, Norwich, NR4 7TJ. Email: c.giebel@uea.ac.uk; Tel.: +44 1603593259

Word count: 3,744 


\section{ABSTRACT}

Background: Minimal evidence exists on the detailed deficits in complex instrumental activities of daily living (IADLs) in mild dementia. The aim of this study was two-fold; to validate a revised questionnaire focusing measuring the initiative and performance of IADLs in mild dementia and to explore the relationship between individual IADLs and patient and carer well-being.

Methods: A total of 183 carers of people with mild dementia completed a further modified Revised Interview for Deterioration in Daily Living Activities 2 (R-IDDD2), which comprised new activities such as computer use, as well as sub-activities on the performance scale. Carers also completed questionnaires assessing patient quality of life (QoL-AD), carer quality of life (AC-QoL) and burden (GHQ-12).

Results: PwD were significantly poorer initiating than performing cleaning, doing repair work and preparing a hot or cold meal, whilst being poorer at performing dressing and following current affairs. Using the computer, preparing a hot meal, finance and medication management were most impaired, whilst more basic activities of dressing, washing oneself, brushing hair or teeth, and preparing a hot drink were most preserved. Poor initiative and performance on nearly all activities was significantly related to reduced carer and patient well-being.

Conclusions: The R-IDDD2 offers a platform to comprehensively assess everyday functioning. Deteriorations in initiative and performance need to be targeted separately in interventions, as the former require effective triggering and the latter structured training and support. Most activities were significantly associated with well-being, particularly patient quality of life, so that improving any activity should improve well-being.

Key words: instrumental activities of daily living; dementia; quality of life; carer burden; initiative; performance 


\section{Introduction}

Losing the motivation or ability to perform everyday activities is one of the primary symptoms of dementia (Giebel et al., 2014; Voigt-Radloff et al., 2012). Everyday activities are categorised into basic activities of daily living (ADLs), including bathing and maintaining continence (Katz et al., 1963), and complex instrumental ADLs (IADLs), including food preparation and shopping (Lawton and Brody, 1969). Of these, IADLs deteriorate to a great extent in the preclinical and early stages of dementia (Mioshi et al., 2007; Verlinden et al., 2015), whilst some ADLs deteriorate to a greater extent in the advanced stages (Giebel et al., 2015). The limitations that IADL and ADL deficits can place upon a person with dementia's (PwD) life, can lead to institutionalisation (Afram et al., 2014) and constitute one of the highest cost factors in dementia care (Jones et al., 2015). Hence promoting independence, well-being and management of dementia post-diagnosis are some of the key priorities of recent governmental initiatives (Alzheimer's New Zealand, 2010; Department of Health, 2009; Norwegian Ministry of Health and Care Services, 2007).

There is little evidence on the detailed deterioration of IADLs in mild dementia (Giebel et al., 2014; Griffith et al., 2003; Peres et al., 2008). The performance of finance management tasks has received most attention in early dementia (Chiong et al., 2014; Griffith et al., 2003; Martin et al., 2013), with available evidence highlighting difficulties with financial concept knowledge, cash transactions, bill payment and cheque book management, amongst others (Griffith et al., 2003; Marson et al., 2001). By understanding which IADLs are mostly impaired, interventions can target these IADLs specifically. In particular, a recent review on the efficacy of cognitive interventions for everyday activities showed that targeting individual activities, rather than global functioning, is more efficacious (Giebel and Challis, 2015).

One important distinction in the assessment of IADL abilities is the intention to perform an activity and the performance itself. In the literature, this distinction is rarely made, and is picked up specifically in the Interview for Deterioration in Daily Living Activities in Dementia (IDDD) (Teunisse et al., 1991). In previous studies on mild and moderate dementia, variations between initiative and performance deficits did not emerge (Teunisse et al., 1997; Voigt-Radloff et al., 2012). By contrast, in a previous examination of mild dementia only, the performance of IADLs was found to be impaired to a greater extent than initiation overall (Giebel et al., 2014). However, one exception to this pattern of deficit lay in the area of social activities, as carers reported PwD as lacking the initiative to engage in 
hobbies or maintain an active social life. It is important to evaluate both aspects of behaviour separately, as PwD may be able to perform an activity but fail to initiate the activity due to a lack of motivation or through forgetting to perform the task. Alternatively, PwD may initiate the task without any prompts, but struggle to complete it successfully. Thus, these two sources of impairment in everyday function can be very distinct from one another and may often require different types of support and interventions, which currently is generally not available.

Limitations in independence impact negatively on the PwD' and carers' well-being (Martyr et al., 2014; Sutcliffe et al., 2016), although little is known as to how individual IADLs are associated with quality of life (QoL) and carer stress. In a previous study employing a mildly modified version of the IDDD, social activities in particular were found to significantly correlate with PwD and carer well-being (Giebel et al., 2014). Indeed, evidence has highlighted that general social activities, such as music, singing groups or art interventions, have a positive effect on PwD QoL (Camic et al., 2013; van der Vleuten et al., 2012). It is thus important to understand which individual activities are associated with well-being, so that interventions for PwD and their carers can directly target those activities to improve overall QoL and reduce levels of stress.

The aims of this study were twofold. The first objective was to validate the new revised IDDD2 (R-IDDD2) in a sample of carers of people with mild dementia, and thus to assess the level of changes in the initiative and performance of IADLs. The second objective was to investigate the relationship between the initiative and performance of IADLs and carer and PwD QoL, as well as carer stress. Based on previous findings (Giebel et al., 2014), it was hypothesised that carers would report more deficits in the performance than the initiative of IADLs. Furthermore, social activities were expected to be negatively associated with carer stress, whilst overall everyday activity deficits were expected to be associated with reduced PwD QoL (Giebel et al., 2014) and reduced carer QoL (Razani et al., 2014). Evidence about specific IADL deficits in the early stages can help in the timely diagnosis of dementia and in the development of interventions targeting initiative and performance deficits using separate approaches. Moreover, interventions can be developed that focus on those activities associated to the greatest extent with well-being, a primary objective of national dementia strategies (Alzheimer Nederland, 2012; Alzheimer's New Zealand, 2010; Department of Health, 2009). 


\section{Methods}

\section{Participants and recruitment}

Carers of people with mild dementia were recruited via convenience sampling through memory clinics of nine NHS trusts across England and through the national research directory Join Dementia Research. Staff and research officers from the Greater Manchester NIHR Clinical Research Network identified suitable participants and distributed the questionnaire. Mild dementia was defined by having a Mini-Mental State Examination (MMSE) (Folstein et al., 1975) score of 21 or above (Earnst et al., 2001), a Montreal Cognitive Assessment (MoCA) (Nasreddine et al., 2005) score of 15 or above, or an equivalent score on the third version of the Addenbrooke's Cognitive Examination (ACE-III) (Hsieh et al., 2013). A lower cut off of 65 on the ACE-III was considered to classify mild dementia. Only carers of PwD were considered whose memory scores were obtained in the past six months.

\section{Measures}

Revised Interview for Deterioration in Daily Living Activities in Dementia 2 (R-IDDD2)

The R-IDDD2 is based on the original IDDD (Teunisse et al., 1991) and on a further adapted version, the R-IDDD (Giebel et al., 2014), and assesses everyday functioning initiative and performance. The original IDDD investigates the initiative to perform (nine items) and the performance (11 items) of individual daily activities, which can be rated on a scale from '0' (never any difficulties) to '4' (always difficulties). Although the IDDD has good construct validity (Voigt-Radloff et al., 2012), the questionnaire has poor concurrent validity and lacks some crucial IADLs such as medication management and transportation. Therefore, Giebel et al. (2014) amended the IDDD further and added activities, including monitoring own day and following familiar routes. Carers were also given the opportunity to elaborate on any difficulties when rating an activity as impaired. This R-IDDD was tested in an interview format with 20 carers of people with mild dementia.

Findings from this pilot study informed the further development of the questionnaire into the R-IDDD2. In particular, carers identified additional activities, such as recognising familiar faces, whilst it also emerged that some existing activities needed to be split into two separate items because responses suggested that two separate activities were subsumed under the one activity the original version. For example, preparing a meal was split into preparing a cold meal and preparing a hot meal; using the telephone/computer was split into both telephone use and computer use; transport was split 
into public transport and driving. Engaging in hobbies and maintaining an active social life were merged into one item as responses on both were similar.

An additional amendment to the R-IDDD was the addition of subcategories for each activity. These subcategories emerged through asking carers in the earlier study (Giebel et al., 2014) how deficits manifested themselves when responding ' 1 ' to ' 4 ' for an activity. In the R-IDDD2, each IADL performance has three subcategories, each of which can be rated from ' 0 ' to ' 4 '. For instance, if a PwD reportedly struggles with finance management, carers are asked to rate performance on the respective three sub-categories of difficulty counting change, sorting out bills and using the cash point. Table 1 shows the detailed break-down of each activity. Some activities may not be relevant to a

$\mathrm{PwD}$, if, for instance, they have never used the computer or managed the finances, the total scores for initiative and performance only consider the number of relevant, or completed, activities. For instance, a sum of 30 on 14 completed or relevant activities suggests a higher level of impairment than a sum of 30 on 20 completed or relevant activities. Therefore, the total score is a percentage score with a higher percentage indicating higher levels of impairments.

\section{General Health Questionnaire (GHQ-12)}

The 12-item GHQ-12 (Goldberg and Williams, 1998) measures recent psychological distress. Items cover the ability to concentrate, confidence, and worthlessness, which are rated on a four-point Likert scale. Participants compare the current level of psychological distress to usual levels, and rate current experiences as similar, greater or lower. A higher score indicates greater psychological distress.

\section{Adult Carer Quality of Life Questionnaire (AC-QoL)}

The 40-item AC-QoL (Elwick et al., 2010) assesses eight areas of carer QoL, including support for caring; caring choice; and carer satisfaction. Each item is rated as 'never', 'some of the time', 'a lot of the time' and 'always' by the carer, which equates to a score between ' 0 ' and ' 3 '. A higher score (maximum 120) indicates better QoL.

Quality of Life in Alzheimer's Disease (QoL-AD)

The QoL-AD (Logsdon et al., 1999) is a reliable measure of PwD QoL either from the PwD or from a proxy perspective (Hoe et al., 2005). For this study, the QoL-AD-Proxy version was employed. The 13 
items on the scale include living situation, physical health, and ability to do things for fun, which are rated from ' 1 ' (poor) to '4' (excellent).

\section{[INSERT TABLE 1]}

\section{Procedure}

Ethical approval was obtained via NRES Ethics Committee North West (14/NW/0241) prior to study begin. Prior to the mail out, six carers provided feedback on the questionnaire design. For this purpose, the researcher (CG) visited the carers in their own homes to be present whilst they completed the questionnaire. This offered the possibility for carers to feed back on the clarity of instructions and colour-scheme, which could be amended before the main send out. Completing the questionnaire took approximately 20 to 30 minutes, and the questionnaire was returned via free-post envelopes. Data were collected from April 2014 to October 2015.

\section{Analysis}

Data were analysed using SPSS 22 for Windows. Demographic characteristics and initiative and performance deficits were assessed using frequency analyses. Paired samples t-tests were used to compare deficits on the initiative and performance of individual activities for PwD. Bivariate correlation analyses were employed to explore the associations between initiative and performance of IADLs and carer burden, carer QoL and PwD QoL. Construct validity of the R-IDDD2 was calculated using Cronbach's alpha. The level to which initiative deficits predicted performance deficits for each activity were analysed using single linear regression analysis. Where carers entered scores on one or all three sub-categories of an activity, but no total score, the median of the sub-category scores was calculated to represent the overall activity score. For hours caring for ADLs, IADLs, or supervising, a maximum of 16 hours per day were employed where carers stated 24 -hour care. This procedure was also used in a previous study (Wuebker et al., 2015). Statistical significance was treated as $p<.05$ across all tests. 


\section{Results}

\section{Carer and PwD characteristics}

In total, 183 carers completed and returned the questionnaire. Table 2 details the demographic characteristics of the sample. Carers were on average $66(+/-11)$ years old and mostly female. Most carers were spouses, lived with the PwD and were their sole carer. Carers spent more time supervising than supporting their relative with ADLs or IADLs. PwD were on average $77(+/-9)$ years old and predominantly male (56.8\%). Most PwD had Alzheimer's disease (61.6\%) which was based on proxy reports and were reported to have shown symptoms for an average of $36(+/-28)$ months, whilst carers had cared for the PwD for an average of $25(+/-25)$ months. The vast majority of PwD lived at home, with very few living in sheltered accommodation.

\section{[INSERT TABLE 2]}

\section{Construct validity of the R-IDDD2}

The internal consistency of the R-IDDD2 was calculated using Cronbach's alpha for initiative and performance of ADLs (washing oneself, dressing, brushing teeth/hair) and IADLs. The R-IDDD2 demonstrated high internal consistency for the initiative of basic ADLs $(\alpha=.894)$; performance of basic $\operatorname{ADLs}(\alpha=.858)$; initiative of complex IADLs ( $\alpha=.934)$; and performance of complex IADLs $(\alpha=.959)$.

\section{Initiative and performance ratings of everyday activities}

Table 3 outlines initiative ratings. Data were normally distributed with data skewness ranging between -.896 and 1.133. Of the 17 activities, using the computer, finance management, and preparing a hot meal were most impaired. In contrast, initiating dressing, washing oneself, preparing a hot drink, and brushing hair or teeth were least impaired. With an overall initiative rating of $45.1+/-25.7$ of dependency, PwD were overall partially independent, with 100 per cent indicating full dependence.

Table 4 outlines the scores on the performance scale. Data were normally distributed with data skewness ranging between -.775 and 1.043. Of the 20 activities, using the computer, managing finances and managing medication were subject to greatest impairment, whilst the majority of PwD were impaired in monitoring a current activity, managing finances, following current affairs, monitoring own day, and engaging in social activities. Few PwD were reported to experience difficulties in performing dressing, washing oneself, brushing hair or teeth, and preparing a hot drink, and 
recognising familiar faces. With an overall performance rating of $46.9+/-27.2$ of dependency, PwD were overall partially independent at performing everyday tasks.

Across both initiative and performance of IADLs, those activities predominantly noted as 'not applicable' were using the computer, driving, and taking public transport. Shopping and preparing a hot meal were also frequently noted as 'not applicable' by female carers of male PwD.

Paired samples t-tests revealed that PwD were significantly less able at performing dressing $(t(170)=-3.593, p \leq .001)$ and following current affairs $(t(171)=-3.195, p<.01)$ than initiating these activities, whilst being significantly less able at initiating cleaning the house/doing repair work $(\mathrm{t}(166)=$ $3.514, p \leq .001)$, preparing a cold meal $(\mathrm{t}(164)=2.097, \mathrm{p}<.05)$, and preparing a hot meal $(\mathrm{t}(154)=$ $3.989, p<.001)$, than on their performance. Variations in medication management approached significance with poorer performance than initiative $(t(166)=-1.877, p=.062)$.

Linear single regression analysis (Table 5) revealed that the initiative of each of the 17 activities covered in both scales significantly predicted the performance of an activity, with between 36.7 percent (using the computer) and 58.9 percent (driving) of variance in the performance explained. In all regression models, PwD gender was controlled for.

\section{[INSERT TABLE 3, 4, AND 5]}

\section{Associations with well-being}

Table 3 also shows the correlation coefficients between initiating activities and well-being. Nearly all activities were significantly associated with well-being, with higher initiative deficits associated with greater carer burden, and reduced carer and PwD QoL. Only using the computer, preparing a hot meal, managing medication, and driving were not associated with carer burden. Preparing a cold meal (and dressing as an ADL) were most strongly related with PwD QoL; brushing teeth or hair with carer burden; and cleaning the house/doing repair work and preparing a cold meal with carer QoL. The associations between activities and the QoL-AD were stronger than those with the AC-QoL or GHQ-12.

Table 4 also shows the correlation coefficients between performing activities and well-being. Performance on every activity was significantly related to well-being, with higher performance deficits associated with increased carer burden and poorer carer and PwD QoL. Engaging in social activities 
(and dressing as an ADL) was related most strongly with PwD QoL; recognising familiar faces with carer burden; and cleaning the house/doing repair work with carer QoL. Performing IADLs was more strongly related to well-being than initiating IADLs.

\section{Discussion}

This study first establishes the R-IDDD2 as a suitable and valid assessment tool for everyday functioning. Using this version of the questionnaire, this study provides new insights into the difficulties of initiating and performing IADLs in mild dementia, and how these are associated with the well-being of PwD and their carers. Several activities were found to be impaired to the greatest extent both in their initiative and performance, including using the computer, finance management, medication management, and driving. Both using the computer and driving are activities which are not included in any other IADL instrument, and are generally assessed by direct observation. To our understanding, no previous studies have compared these two activities alongside others, particularly with regard to measuring initiative to engage. In comparison to using transport, telephoning, and medication management, one study showed that finance management impairments at baseline were a significant predictor of developing dementia 10 years later (Peres et al., 2008). Difficulties in medication management have also been reported to be amongst the most prominent (Giebel et al., 2014), although research mostly fails to distinguish between medication management and keeping appointments (Brown et al., 2011; Martyr et al., 2012). Therefore the R-IDDD2, and its use in the current study, provide a more detailed assessment of individual IADL difficulties than previous tools and their application have allowed.

Consistent with our hypothesis, some activities showed significant variations between their initiative and performance, although most activities reported similar impairment across the two measures. Whilst multiple tests of comparison with the initiative and performance might have increased the Type I error, the purpose of this study was to provide a first validation of the R-IDDD2, and applying Bonferroni correction to the analyses would have increased the Type II error considering the number of tests of comparisons performed. The variations evidenced here can have important implications for the effective management of dementia. Most non-pharmacological interventions on everyday functioning focus on global IADLs or ADLs, or on some selected activities (Clare et al., 2000; Giebel and Challis, 2015; Kurz et al., 2009). These fail to distinguish between the initiative and 
performance of activities. However, findings from this study distinguishing between initiative and performance deficits, suggest that PwD might either need better 'triggers' (to encourage initiative) or better training or support (to improve performance) in order to ensure that functioning and independent living are optimised. Across the sample, cleaning/doing repair work and preparing a hot or cold meal were impaired to a greater extent in their initiative than their performance. This suggests that interventions targeting structured training by teaching, for example the individual steps of those activities, would prove minimally effective. Instead, PwD need to be provided with effective triggers, in order to help initiate those tasks. This could be achieved by employing simple memory aids, such as specific alarms (Gibson et al., 2015) or maybe simply by using lists and signs. In contrast, for dressing, following current affairs, and medication management, triggers would be less effective, as the performance is impaired to a greater extent. Thus, PwD require structured training to improve performance on the individual steps of those tasks. This might be achieved by structured cognitive interventions, which could address the specific cognitive deficits underpinning the impairment in performance of activities. It is worth noting that patterns of everyday functioning deficits in PwD can vary considerably across individuals, even at the early stages of dementia. Therefore, the approach of using either triggers or training needs to be adapted to the individual IADL deficits of PwD.

Overall, there appears to be no clear message as to whether initiative or performance deficits are more prominent in early dementia. This corroborates previous evidence obtained with mild-tomoderate dementia populations (Teunisse et al., 1991; Voigt-Radloff et al., 2012). One possibility for not finding clearer distinctions between initiative and performance could be the presence of significant individual differences across PwD as mentioned above. Old age and its increased likelihood of comorbidities might have been a biasing factor in this study, considering also that PwD were on average 10 years older than carers. Furthermore, levels of everyday functioning are associated with depression, physical limitations, differing cognitive deficits and environmental modifications (Fauth et al., 2013; Liu-Seiffert et al., 2015). These were not assessed in this study, so as to not overburden carers, and hence, these unexplained factors may have played a role in IADL activity. Future research should investigate how these potentially biasing factors might differentially influence initiative and performance.

In support of our second hypothesis, the majority of IADLs were strongly associated with the well-being of both carers and PwDs. Engaging in social activities was particularly strongly associated 
with PwD QoL, consistent with previous evidence (Giebel et al., 2014), suggesting that the loss of one's capacity for social engagement has a particularly negative effect on the well-being of PwD. The majority of studies employ proxy reports for everyday functioning and/ or well-being (Beerens et al., 2015; Mioshi et al., 2009), similar to this study, so that future research should evaluate whether there are variations in PwD perspectives of well-being. Although nearly all activities were significantly associated with well-being, it would be interesting to explore this relationship further in the more advanced stages of dementia. As PwD and carers become more accommodated to their new circumstances and caring roles, it might be the case that other activities impact on the well-being than those in the initial early stages. This is based on the hierarchy of daily activity decline reported first by Reisberg and colleagues (Reisberg et al., 1984).

\section{Conclusions}

This study established the R-IDDD2 as a comprehensive tool that evaluates several layers of everyday functioning. Its application here provides novel insights into impairments in initiating and performing IADLs in mild dementia, and how these could be effectively managed. As opposed to previous scales (i.e. Lawton and Brody, 1969; Sikkes et al., 2012), the purpose of the R-IDDD2 is to focus on individual activities and sub-activities. Given this level of detail, the R-IDDD2 appears to be a suitable, and internally consistent, tool in capturing subtle changes in everyday functioning, which might not only help in the diagnosis of very mild dementia but also in possibly identifying specific deficits suitable for interventions. Findings from this study suggest that interventions to improve functioning need to address initiative and performance deficits in a targeted way, by either employing triggers or providing structured training or support. Future research should also explore how wellbeing is related to individual IADLs in more advanced stages of dementia and how specific subtypes of dementia may experience different levels of impairments.

\section{Conflict of interest}

None. 


\section{Funding Sources}

This article presents independent research supported by the National Institute for Health Research (NIHR), under its Programme Grants for Applied Research programme (Grant Reference Number: DTC-RP-PG-0311-12003), and forms part of the doctoral thesis of CG.

\section{Description of author roles}

CG collected the data, performed statistical analysis, and wrote the manuscript. CG and DM designed the study. DM and DC provided feedback to drafts of the manuscript.

\section{Acknowledgements}

We wish to thank staff at the following NHS Trusts for their support in recruiting participants: Catherine Turton, Joanne Woodward at 5 Boroughs Partnership; Ritchard Ledgerd, Alexandra Feast, Charlotte Stoner, Dr Joanne Rodda at North East London; Paula Harman, Dr Farhad Huwez at Southend University Hospital; Joanne Tomkins, Laura Lord, Dr Tarik Qassem at Black Country Partnership; Olga Balazikova, Sarah Hamilton, Ramin Nilforooshan at Surrey and Borders Partnership; Lisa WilkinsonGuy, Prof George Tadros at Heart of England; and Kim Thompson, Leigh Franks, Dr Rashi Negi at South Staffordshire and Shropshire Healthcare. We also wish to thank Division 4 Greater Manchester NIHR Clinical Research Network for recruiting participants to this study through Manchester Mental Health and Social Care and Greater Manchester West NHS Trusts. Our thanks also extend to the JoinDementiaResearch Network for allowing us to recruit carers through their network. 


\section{References}

Afram, B., et al. (2014). Reasons for Institutionalization of People With Dementia: Informal Caregiver Reports From 8 European Countries. Journal of the American Medical Directors Association, 15(2), 108-116.

Alzheimer Nederland (2012). DeltaPlan Dementie. Alzheimer Nederland.

Alzheimers New Zealand (2010). National Dementia Strategy 2010-2015. Wellington.

Ballard, C., Burns, A., Corbett, A., Livingston, G. and Rasmussen, J. (2013). Helping you to assess cognition: A practical toolkit for clinicians. London: Alzheimer's Society.

Beerens HC, et al. (2015). Change in quality of life of people with dementia recently admitted to longterm care facilities. Journal of Advanced Nursing, 71(6), 1435-1447.

Brown, P.J., Devanand, D.P., Liu X, Caccappolo E. (2011). Functional impairment in elderly patients with mild cognitive impairment and mild Alzheimer disease. Archives of General Psychiatry, 68(6), 617-626.

Camic P, Williams CM, Meeten F (2013). Does a 'Singing Together Group' improve the quality of life of people with dementia and their carers? A pilot evaluation study. Dementia, 12(2), 157-176.

Chiong W, Hsu M, Wudka D, Miller BL, Rosen HJ. (2014). Financial errors in dementia: Testing a neuroeconomic conceptual framework. Neurocase, 20(4), 389-396.

Clare L, et al. (2000). Intervening with everyday memory problems in dementia of Alzheimer type: an errorless learning approach. Journal of Clinical and Experimental Neuropsychology, 22, 132-146.

Clarke, D.E., et al. (2008). Apathy in Dementia: Clinical and Sociodemographic Corrrelates. Journal of Neuropsychiatry and Clinical Neuroscience, 20, 337-347.

Department of Health (2009). Living well with dementia: A National Dementia Strategy. Accessible Summary. London: Department of Health.

Earnst KS, Wadley VG, Aldridge TM, Steenwyk AB, Hammond AE, Harrell LE, Marson DC (2001). Loss of financial capacity in Alzheimer's disease: The role of working memory. Aging, Neuropsychology, and Cognition, 8(2), 109-119.

Elwick H, Joseph S, Becker S, Becker F (2010). Manual for the Adult Carer Quality of Life Questionnaire $(A C-Q O L)$. London: The Princess Royal Trust for Carers.

Fauth EB, Schwartz S, Tschanz JT, Ostbye T, Corcoran C, Norton MC (2013). Baseline disability in activities of daily living predicts dementia risk even after controlling for baseline global cognitive ability and depressive symptoms. International Journal of Geriatric Psychiatry, 28(6), 597-606.

Fieo R, Manly JJ, Schupf N, Stern Y (2014). Functional Status in the Young-Old: Establishing a Working Prototype of an Extended-Instrumental Activities of Daily Living Scale. Journal of Gerontology Medical Sciences, 69(6), 766-772.

Folstein M, Folstein S, McHugh P (1975). "Mini-mental state". A practical method for grading the cognitive state of patients for the clinician. Journal of Psychiatric Research, 12, 189 - 198.

Gelinas I, Gauthier L, McIntyre M, Gauthier S (1999). Development of a functional measure for persons with Alzheimer's disease: the disability assessment for dementia. American Journal of Occupational Therapy, 53, 471-481.

Gibson G, Dickinson C, Brittain K, Robinson L (2015). The everyday use of assistive technology by people with dementia and their family carers: a qualitative study. BMC Geriatrics, 15, 89.

Giebel C, Challis, D (2015). Translating cognitive and everyday activity deficits into cognitive interventions in mild dementia and mild cognitive impairment. International Journal of Geriatric Psychiatry, 30(1), 63-71.

Giebel CM, Challis D, Montaldi D (2014). A revised Interview for Deterioration in Daily Activities in Dementia (R-IDDD) reveals the relationship between social activities and wellbeing. Dementia, doi: $10.1177 / 1471301214553614$

Giebel CM, Sutcliffe C, Challis D (2015). Activities of Daily Living and Quality of Life across different Stages of Dementia: A UK Study. Aging Ment Health,19(1), 63-71.

Goldberg, D. and Williams, P (1998). A user's guide to the General Health Questionnaire. Windsor, UK: NFER-Nelson.

Hoe J, Katona C, Roch B, Livingston G (2005). Use of the QoL-AD for measuring quality of life in people with severe dementia - the LASER-AD study. Age and Ageing, 34:130-135.

Jones RW, Romeo R, Trigg R, Knapp M, Sato A, King D, Niecko T, Lacey L (2015). Dependence in Alzheimer's disease and service use costs, quality of life, and caregiver burden: The DADE study. Alzheimer's \& Dementia,11(3), 280-290.

Katz S, Ford AB, Moskowitz RW, Jackson BA, Jaffe MW (1963). Studies of illness in the aged: The index of ADL: a standardized measure of biological and psychosocial function. Journal of the 
American Medical Association, 185(12), 914-919.

Kurz A, Pohl C, Ramsenthaler M, Sorg C (2009). Cognitive rehabilitation in patients with mild cognitive impairment. International Journal of Geriatric Psychiatry, 24, 163-168.

Lawton MP, Brody EM (1969). Assessment of older people: Self-maintaining and instrumental activities of daily living. Gerontolology, 9(3), 179-186.

Liu-Seiffert H, Siemers E, Sundell K, Price K, Han B, Selzler K, Aisen P, Cummings J, Raskin J, Mohs R (2015). Cognitive and Functional Decline and Their Relationship in Patients with Mild Alzheimer's Dementia. Journal of Alzheimer Disease, 43(3), 949-955.

Logsdon RG, Gibbons LE, McCurry SM, Teri L (1999). Quality of life in Alzheimer's disease: Patient and caregiver reports. Journal of Mental Health and Aging, 5(1), 21-32.

Marson DC, et al. (2000). Assessing financial capacity in patients with Alzheimer's disease: A conceptual model and prototype instrument. Archives of Neurology, 57, 877-884.

Martin RC, et al. (2013). Impaired financial abilities in Parkinson's disease patients with mild cognitive impairment and dementia. Parkinsonism and Related Disorders, 19(11), 986-990.

Martyr A, et al. (2012). Verbal Fluency and Awareness of Functional Deficits in Early-Stage Dementia. Clinical Neuropsychologist, 26(3), 501-519.

Martyr A, Nelis SM, Clare L (2014). Predictors of perceived functional ability in early-stage dementia: self-ratings, informant ratings and discrepancy scores. International Journal of Geriatric Psychiatry, 29(8), 852-862.

Mioshi E, Bristow M, Cook R, Hodges JR (2009). Factors Underlying Caregiver Stress in Frontotemporal Dementia and Alzheimer's Disease. Dementia and Geriatric Cognitive Disorders, 27, 76-81.

Mioshi E, Kipps CM, Dawson K, Mitchell J, Graham A, Hodges JR (2007). Activities of daily living in frontotemporal dementia and Alzheimer disease. Neurology, 68(24), 2077-2084.

Norwegian Ministry of Health and Care Services (2007). Dementia Plan 2015. Norway: Norwegian Ministry of Health and Care Services.

Nasreddine ZS, Phillips NA, Bedirian V, Charbonneau S, Whitehead V, Collin I, Cummings JL, Chertkow H (2005). The Montreal Cognitive Assessment, MoCA: a brief screening tool for mild cognitive impairment. Journal of the American Geriatrics Society, 53(4), 695-699.

Peres K, Helmer C, Amieva H, Orgogozo JM, Rouch I, Dartigues JF, Barberger-Gateau P (2008). Natural history of decline in instrumental activities of daily living performance over the 10 years preceding the clinical diagnosis of dementia: A prospective population-based study. Journal of the American Geriatrics Society, 56(1), 37-44.

Razani J, Corona R, Quilici J, Matevosyan AA, Funes C, Larco A, Miloyan B, Avila J, Chung J, Goldberg H, Lu P (2014). The Effects of Declining Functional Abilities in Dementia Patients and Increases in Psychological Distress on Caregiver Burden Over a One-Year Period. Clinical Gerontology,37(3), 235-252.

Reisberg B, Ferris SH, Anand R, de Leon MJ, Schneck MK, Buttinger C, Borenstin J (1984). Functional staging of dementia of the Alzheimer's type. Annals of the New York Academy of Sciences, 435, 481-483.

Sikkes SAM, de Lange-de Klerk ES, Pijnenburg YA, Gillissen F, Romkes R, Knol DL, Uitdehaag BMJ, Scheltens P (2012). A new informant-based questionnaire for instrumental activities of daily living in dementia. Alzheimer's and Dementia, 8, 536-543.

Sutcliffe CL, Giebel CM, Jolley D, Challis D (2016). Experience of burden in carers of people with dementia at the margins of long-term care. International Journal of Geriatric Psychiatry, 31(2), 101-108.

Teunisse, S., Derix, M.M.A. and van Crevel, H. (1991). Assessing the severity of dementia. Archives of Neurology, 48(3), 274-277.

Teunisse, S. and Derix, M.M. (1997). The interview for deterioration in daily living activities in dementia: agreement between primary and secondary caregivers. International Psychogeriatrics, 9(1), 155-162.

van der Vleuten, M., Visser, A. and Meeuwesen, L. (2012). The contribution of intimate live music performances to the quality of life for persons with dementia. Patient Education and Counselling, 89(3), 484-488.

Verlinden, V.J.A., van der Geest, J.N., de Bruijn, R.F.A.G., Hofman, A., Koudstaal, P.J. and Arfan Ikram, M. (2015). Trajectories of decline in cognition and daily functioning in preclinical dementia. Alzheimer's and Dementia, doi: 10.1016/j.jalz.2015.08.001

Vermeersch, S., Gorus, E., Cornelis, S. and De Vriendt, P. (2015). An explorative study of the relationship between functional and cognitive decline in older persons with mild cognitive impairment and Alzheimer's disease. British Journal of Occupational Therapy, 78(3), 166-174. 
Voigt-Radloff, S., et al. (2012). Interview for Deterioration in Daily Living Activities in Dementia: construct and concurrent validity in patients with mild to moderate dementia. International Psychogeriatrics, 24(3),382-390.

Wübker, A., et al. (2015). Costs of care for people with dementia just before and after nursing home placement: primary data from eight European countries. European Journal of Health Economics, 16, 689-707. 